\section{Análise comparativa dos sistemas de saúde da tríplice fronteira: Brasil/Colômbia/Peru}

\author{
Antonio Levino ${ }^{1}$ e \\ Eduardo Freese de Carvalho
}

Como citar: Levino A, Carvalho EF. Análise comparativa dos sistemas de saúde da tríplice fronteira: Brasil/Colômbia/Peru. 2011;30(5):490-500.

\section{RESUMO}

Os objetivos deste artigo são: descrever o processo de reforma sanitária ocorrido em países da América Latina e suas repercussões nos sistemas de saúde do Brasil, da Colômbia e do Peru; analisar a conformação dos sistemas nacionais de saúde desses três países fronteiriços; e avaliar os limites e possibilidades de uma integração dos sistemas locais de saúde no contexto da tríplice fronteira amazônica. A partir de documentos $e$ bases de dados oficiais, faz-se uma análise do desenvolvimento dos sistemas de saúde e da organização da rede de serviços nesse segmento de fronteira localizado em plena região amazônica. Na comparação entre os três países, constata-se que o modelo adotado pelo Brasil tem as características de um sistema nacional de saúde de finalidade pública, enquanto na Colômbia e no Peru vigoram sistemas de asseguramento, com adoção de planos obrigatórios de assistência, segmentação dos usuários e contingenciamento da cobertura. Os três países convergem quanto à mescla de público e privado na provisão de serviços, mas diferem no nível de acesso e no tipo de regulação por parte do Estado. Conclui-se que as especificidades dos sistemas de saúde dos três países fronteiriços tornam complexo o desafio da construção de um sistema único de saúde na fronteira que possa garantir a universalidade do acesso, a equidade no atendimento e a integralidade da assistência. Apesar das discrepâncias identificadas, porém, constata-se que a preponderância do setor público na rede de atenção básica permite vislumbrar a possibilidade de integração dos sistemas locais de saúde no que concerne à oferta organizada

1 Instituto Leônidas e Maria Deane, Fundação Oswaldo Cruz ILMD/FIOCRUZ, Manaus, Amazonas, Brasil. Correspondência: antoniolevino@ibest.com.br

2 Centro de Pesquisas Aggeu Magalhães - CPqAM/FIOCRUZ Recife, Pernambuco, Brasil. de ações programáticas de saúde do primeiro e segundo níveis de assistência.

Palavras-chave: sistemas de saúde; administração de serviços de saúde; reforma dos serviços de saúde; saúde na fronteira; Brasil; Colômbia; Peru; América do Sul.

O estado de saúde em regiões de fronteira aberta sofre grande influência da mobilidade populacional, que incrementa o risco da emergência e reemergência de doenças, e das condições de vida de grupos mais vulneráveis como: migrantes; minorias étnicas; trabalhadores que realizam atividades temporárias, tais como madeireiros e garimpeiros; turistas; ou, ainda, profissionais do sexo. Nessas áreas, a assistência à saúde é sempre complexa, pois mesmo quando a capacidade instalada permite o acolhimento das demandas a efetividade das ações pode ser comprometida pelo fluxo dos usuários que buscam superar, individualmente, as carências do seu país de origem (1).

Este artigo analisa as características do sistema de saúde da tríplice fronteira Brasil/Colômbia/Peru, ${ }^{3}$ tomando por referência a política de saúde dos três países fronteiriços (figura 1). Para tal finalidade, a depender da abordagem, Viacava (3) sugere o uso dos termos "sistema de saúde" e "sistema de serviços de saúde". O primeiro contempla os determinantes históricos e estruturais das concepções sobre saúde/adoecimento e do modo de intervir nesses processos; o segundo é entendido como um conjunto de atividades cujo principal propósito seria o de promover, restaurar e manter a saúde, constituindo uma resposta organizada às necessidades, demandas e representações de uma dada sociedade em determinada época $(4,5)$.

A categoria "sistema de saúde" serve então para elucidar as relações entre este setor e os seus determinantes externos, sem dar conta, porém, da singularidade dos processos, porque não existe um "sistema" que se reproduz em tempos e lugares diversos. O que se reconhece são generalidades ou "sistemas" que expressam as nuanças contextuais na organização

\footnotetext{
A fronteira internacional do Brasil (2) é subdividida em três grandes arcos: o Arco Norte, que compreende a faixa de fronteira dos estados do Amapá, Pará, Amazonas e os estados de Roraima e Acre; o Arco Central, que inclui os estados de Rondônia, Mato Grosso e Mato Grosso do Sul; e o Arco Sul, que compreende a fronteira dos estados do Paraná, Santa Catarina e Rio Grande do Sul. No caso do Arco Norte, segundo a política oficial do governo brasileiro, as diferenças na base produtiva, a posição geográfica e o predomínio de população indígena foram considerados como critérios para a definição das sub-regiões, que são identificadas como: Oiapoque-Tumucumaque (I), Campos do Rio Branco (II), Parima-Alto Rio Negro (III), Alto Solimões (IV), Alto Juruá (V) e Vale do Acre-Alto Purus (VI).
} 
FIGURA 1. Tríplice fronteira Brasil-Colômbia-Peru. Destaque para as áreas fronteiriças do estado do Amazonas (BR) e dos departamentos do Amazonas (CO) e Loreto (PE)

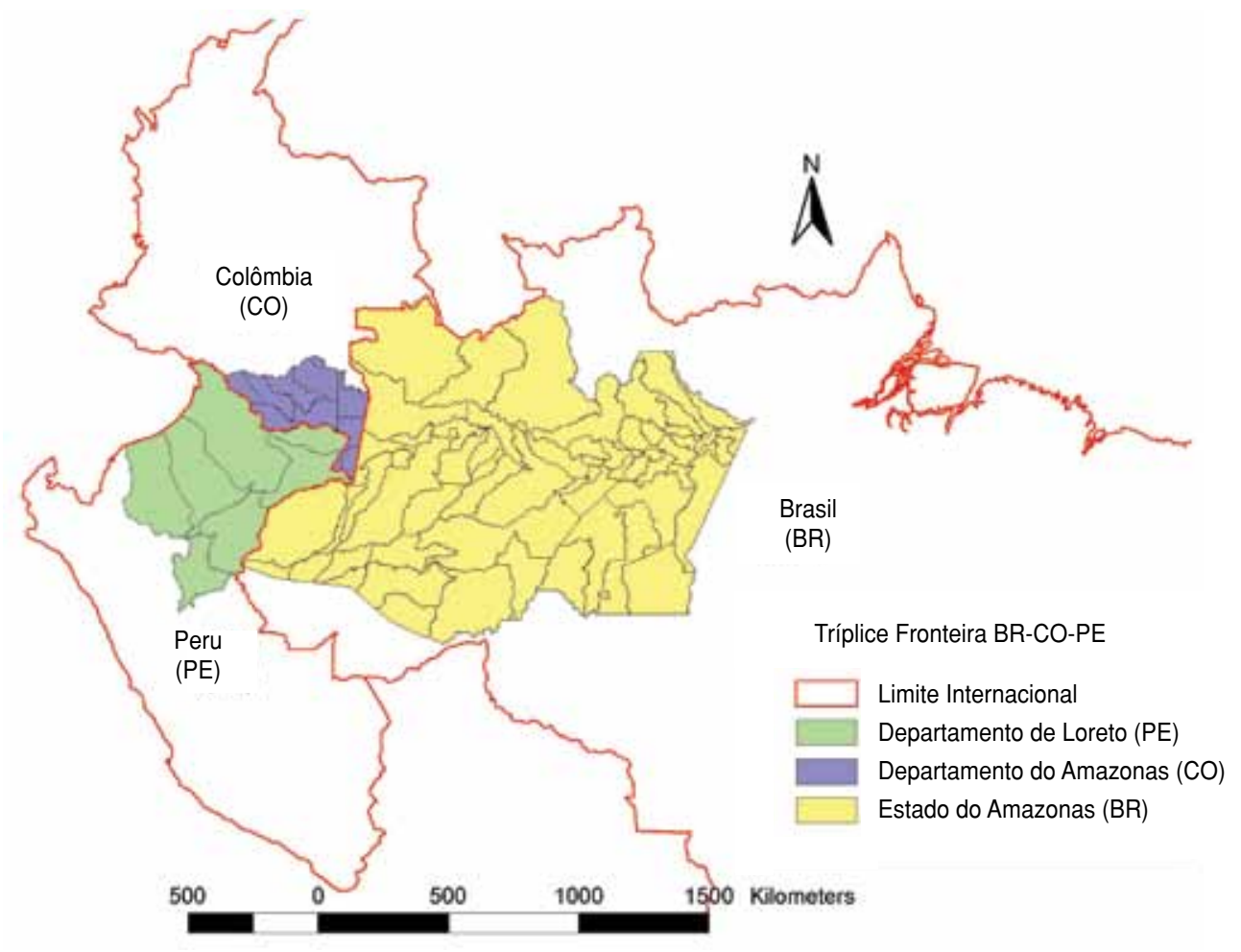

Fonte: Elaborada pelos autores

dos sistemas de serviços de saúde, que resultam da experiência e cultura sanitária de cada sociedade. $\mathrm{Na}$ análise comparada dos sistemas nacionais de saúde é possível identificar convergências e divergências entre as diferentes estratégias adotadas na solução de problemas comuns, como ocorre na tríplice fronteira Brasil/ Colômbia/Peru.

A tríplice fronteira amazônica abrange a divisa internacional que separa o Estado do Amazonas, no Brasil, dos departamentos do Amazonas na Colômbia e Loreto no Peru. A extensão da fronteira entre o Brasil e a Colômbia mede 1645 quilômetros, entre o Brasil e o Peru mede 1 154,30 quilômetros e entre a Colômbia e o Peru mede 1626 quilômetros (figura 1). O Estado do Amazonas representa 18,40\% do território brasileiro e subdivide-se em 62 municípios que somam 3393369 habitantes, dos quais 186281 (5,48\%) estão localizados no Alto Solimões, que abrange sete municípios (tabela 1). Esta sub-região é considerada uma das áreas mais críticas da Amazônia brasileira, por concentrar os principais determinantes dos problemas de saúde que são comuns às zonas de fronteira: elevada mobilidade populacional, atividades de grande impacto ambiental, ocupação desordenada do espaço, falta de acesso aos serviços de saúde e condição de vida precária de determinados grupos populacionais, o que tem como consequência direta uma intensificação das relações transfronteiriças $(1,6,7)$.
O Amazonas colombiano abrange 9,60\% do território nacional, sendo composto por dois municípios (Leticia e Puerto Nariño) e nove corregimientos, territórios pouco povoados e com baixo desenvolvimento econômico e social. Dos seus 71167 habitantes, mais da metade $(65,73 \%)$ está concentrada na capital Leticia que, juntamente com Puerto Nariño, ultrapassa 60\% do total da população departamental (tabela 1) (8). O Departamento de Loreto ocupa $28 \%$ do território do Peru, sendo composto por sete províncias e 51 distritos. Sua população estimada é de 970928 habitantes (tabela 1). Em termos populacionais, Iquitos, a capital do departamento, é considerada a principal cidade de Loreto, mas, do ponto de vista das relações transfronteiriças entre o Brasil, Colômbia e Peru, Caballococha é a cidade mais importante, em função do intenso comércio e por servir de referência para as localidades de Santa Rosa e Islândia, situadas na proximidade de Leticia (CO), Tabatinga (BR) e Benjamin Constant (BR) (9).

Os objetivos do artigo são: descrever o processo de reforma sanitária ocorrido em países da América Latina e suas repercussões nos sistemas de saúde do Brasil, da Colômbia e do Peru; analisar a conformação dos sistemas nacionais de saúde desses três países fronteiriços; e avaliar os limites e possibilidades de uma integração dos sistemas locais de saúde no contexto da tríplice fronteira amazônica. 
TABELA 1. Unidades territoriais do Brasil, Colômbia e Peru que formam a tríplice fronteira amazônica, segundo a extensão territorial e distribuição da população

\begin{tabular}{|c|c|c|c|c|}
\hline Unidades territoriais & $\begin{array}{l}\text { Extensão } \\
\left(\mathrm{km}^{2}\right)\end{array}$ & $\begin{array}{l}\text { População } \\
\text { (2009) }\end{array}$ & $\begin{array}{l}\text { População } \\
\%\end{array}$ & $\begin{array}{l}\text { Densidade } \\
\left(\mathrm{hab} / \mathrm{km}^{2}\right)\end{array}$ \\
\hline \multicolumn{5}{|l|}{$\begin{array}{l}\text { Brasil: municípios da região do } \\
\text { Alto Solimões }\end{array}$} \\
\hline Amaturáa & 4758 & 8828 & 4,74 & 1,86 \\
\hline Atalaia do Norte ${ }^{a}$ & 76354 & 14715 & 7,89 & 0,19 \\
\hline Benjamin Constant ${ }^{a}$ & 8793 & 31195 & 16,75 & 3,55 \\
\hline Santo Antônio do lçáa & 12307 & 30351 & 16,29 & 2,47 \\
\hline São Paulo de Olivençaa & 19745 & 32958 & 17,69 & 1,67 \\
\hline Tabatinga $^{a}$ & 3225 & 47948 & 25,74 & 14,87 \\
\hline Tonantins $^{a}$ & 6432 & 20286 & 10,89 & 3,15 \\
\hline Total & 131614 & 186281 & 100,00 & 1,42 \\
\hline \multicolumn{5}{|c|}{$\begin{array}{l}\text { Colômbia: municípios e corregimientos } \\
\text { do departamento do Amazonas }\end{array}$} \\
\hline El Encanto & 11074 & 4570 & 6,42 & 0,41 \\
\hline La Chorrera & 12461 & 3554 & 4,99 & 0,29 \\
\hline La Pedrera ${ }^{a}$ & 15385 & 4193 & 5,89 & 0,27 \\
\hline La Victoria & 1443 & 1029 & 1,45 & 0,71 \\
\hline Leticia $^{a}$ & 5829 & 39317 & 55,25 & 6,75 \\
\hline Miriti-Paraná & 16564 & 1587 & 2,23 & 0,10 \\
\hline Puerto Alegría ${ }^{a}$ & 8394 & 1513 & 2,13 & 0,18 \\
\hline Puerto Arica ${ }^{a}$ & 13350 & 1408 & 1,98 & 0,11 \\
\hline Puerto Nariñoa & 1475 & 7456 & 10,48 & 5,05 \\
\hline Puerto Santander & 14915 & 2590 & 3,64 & 0,17 \\
\hline Tarapacáa & 8775 & 3950 & 5,55 & 0,45 \\
\hline Total & 109665 & 71167 & 100,00 & 0,65 \\
\hline \multicolumn{5}{|l|}{$\begin{array}{l}\text { Peru: províncias do } \\
\text { departamento de Loreto }\end{array}$} \\
\hline Alto Amazonas & 18764 & 114089 & 11,75 & 6,08 \\
\hline Datem del Marañon & 46609 & 54170 & 5,58 & 1,16 \\
\hline Loreto & 67434 & 69133 & 7,12 & 1,03 \\
\hline Maynas $^{a}$ & 119859 & 534557 & 55,06 & 4,46 \\
\hline Ramón Castillaa & 37412 & 60529 & 6,23 & 1,62 \\
\hline Requena $^{\mathrm{a}}$ & 49477 & 71686 & 7,38 & 1,45 \\
\hline Ucayali & 29293 & 66764 & 6,88 & 2,28 \\
\hline TOTAL & 350087 & 970928 & 100,00 & 2,77 \\
\hline
\end{tabular}

Fontes: Brasil: DATASUS e IBGE (Censos e estimativas); Colômbia: Proyecciones de población 2005-2020 http://www.dane.gov.co/daneweb V09/index.php?option=com content\&view=article\&id=75\&ltemid=72[acessadoem 13/05/2010]; Peru: Dirección Regional de Salud Loreto (DIRESA) MINSA http://www.minsa.gob.pe/estadisticas/estadisticas/Poblacion/ PoblacionMarcos asp?16 [acessado em 13/05/2010].

a Áreas situadas na zona da tríplice fronteira Brasil/Colômbia/Peru.

\section{Síntese do processo de reforma sanitária na América Latina}

Praticamente todos os países da América Latina e do Caribe passaram por reformas setoriais no âmbito da saúde. São processos recentes, ainda inconclusos e marcados por contradições relacionadas ao conteúdo dos projetos e aos resultados alcançados. Sua principal motivação foram as crises que se abateram sobre os sistemas de saúde da região, manifestadas pelo acesso restrito a serviços de qualidade precária e custos elevados (10). No cenário internacional, o neoliberalismo e a globalização também formaram o pano de fundo de uma série de mudanças na ordem política e econômica que provocaram a redução do papel do Estado, uma premissa que passou a ser adotada como um paradigma nas relações de produção, circulação e consumo de mercadorias, serviços e capital (11-14). É nesse contexto que as reformas sanitárias enfrentaram os problemas de financiamento e de gestão do sistema de saúde com resultados distintos em cada país, dependendo do nível de estruturação das políticas públicas, da correlação de forças interna dos grupos políticos dominantes e do grau de interação da comunidade técnico-científica com as estruturas de poder no âmbito dos governos $(10,11)$.

De acordo com o modelo de reforma adotado, consolidaram-se dois pólos. Nos países onde a concepção de Estado mínimo foi assimilada, a saúde passou a ser vista como uma mercadoria e o usuário como consumidor. Nestes, o acesso aos serviços se dá pelo asseguramento ou desembolso direto, nos moldes do que vigora nos Estados Unidos. Nos países onde se manteve o princípio da solidariedade e o conceito de 
cidadania, foram estruturados modelos, como o Sistema Único de Saúde (SUS) do Brasil, que garantem o acesso universal e a assistência integral. Entre os dois extremos formaram-se modelos intermediários, que variam no nível de acesso e amplitude da cobertura, assim como nas modalidades de afiliação aos seguros e no grau de integração entre o setor público e o setor privado para a prestação de serviços $(14,15)$.

Segundo García (15), apesar de não se ter produzido uma agenda específica para o setor da saúde, o consenso neoliberal em torno de um ajuste estrutural da economia continha alguns pontos que foram assimilados como diretrizes nos projetos de reforma sanitária. Para Gómez-Camelo (10), esses delineamentos estão explicitados em dois documentos do Banco Mundial: "Financing Health Services in Developing Countries: An Agenda for Reform", de 1987, e no "Informe Sobre el Desarrollo Mundial 1993: Invertir en Salud".

Mesmo sem lograr êxito na promessa de reduzir as iniquidades em saúde, melhorar o uso eficiente dos recursos e aumentar a qualidade dos serviços, o Banco Mundial continua influenciando a tendência de padronização dos sistemas de saúde com premissas que García (15) resume em quatro eixos de intervenção: a) contenção do crescimento dos investimentos no setor saúde; b) separação das funções de provisão e financiamento; c) focalização do financiamento público somente para grupos vulneráveis; e d) racionamento da assistência médica. As contradições entre esses delineamentos externos e os interesses nacionais repercutiram de modo peculiar na reforma sanitária do Brasil, da Colômbia e do Peru.

\section{Constituição dos sistemas de saúde do Brasil, da Colômbia e do Peru}

Brasil

A reforma sanitária brasileira nasceu na década de 1980, como desdobramento de um debate amplo que envolveu a sociedade civil organizada, a comunidade técnico-científica e a esfera governamental, resultando na proposta do SUS incorporada à plataforma política do movimento que defendia a redemocratização do país. Contrariando a tendência neoliberal, os preceitos do novo sistema de saúde foram introduzidos na constituição promulgada em 1988 (16), passando a ser reconhecidos como um direito de todos: a universalidade do acesso, a equidade no atendimento e a integralidade da assistência. Posteriormente, a Lei Orgânica da Saúde - LOS 8080/1990 e a Lei Complementar 8142/1990 $(17,18)$ estabeleceram as bases operacionais definitivas para o processo de descentralização, hierarquização e controle social previstos no texto constitucional (19).

A mudança no arcabouço jurídico institucional exigiu uma ampla redefinição das atribuições e dos papéis dos diferentes níveis de governo em relação à saúde (20). O SUS prevê a descentralização do planejamento e execução das ações para a esfera municipal, que gradativamente tem assumido a responsabilidade pela organização das redes locais de atenção, respeitando os níveis de complexidade dos problemas. Nas áreas em que dispõe de competência, o setor público assume diretamente a cobertura da assistência lançando mão de serviços próprios que podem ser vinculados a qualquer uma das três esferas de governo: federal, estadual ou municipal.

A rede privada passou a ser tratada como um sistema complementar, que pode ser demandado pelo poder público para a realização de exames diagnósticos ou assistência de média e alta complexidade, principalmente hospitalar, nas áreas em que não se dispõe de uma estrutura própria do governo, sendo todos esses procedimentos cobertos pelos recursos públicos. Mais recentemente, a gestão do SUS tem sido reorientada no sentido da regionalização da assistência, definindo territórios sanitários de abrangência supramunicipal. Para viabilizar as ações, os recursos financeiros são repassados diretamente aos municípios, que devem elaborar um plano de metas prevendo mudanças efetivas na situação de saúde da população (21).

O financiamento do sistema se dá pelos fundos de saúde de nível nacional, estadual e municipal, que são supridos por recursos orçamentários ou oriundos da arrecadação fiscal e previdenciária de empregados e empregadores, contribuições sociais vinculadas à lucratividade das empresas e dos concursos de prognósticos (loterias), além da cooperação internacional (20). As transferências entre esferas ocorrem mediante: repasses fundo a fundo, com base no critério populacional; convênios vinculados às ações prioritárias; ou, ainda, pagamento de procedimentos realizados diretamente às entidades prestadoras de serviços (21).

\section{Colômbia}

A conformação atual do sistema de saúde da Colômbia tem origem no pacto nacional pela superação da crise institucional desencadeada pelo conflito com o narcotráfico entre 1991 e 1993. A constituição promulgada em 1991 incorporou a noção de Estado social de direito, garantindo o acesso universal à seguridade social; entretanto, a tradição colombiana de promover reformas amplas para obter a distensão política foi mantida no espírito da Lei 100/1993 (22) que transformou o Sistema Nacional de Saúde (SNS) em Sistema Geral de Seguro Social em Saúde (SGSSS). Para evitar o confronto com o sindicalismo e ao mesmo tempo garantir o apoio dos setores conservadores ao projeto de abertura da economia do país, o governo conduziu a reforma de maneira que o novo sistema preservasse a lógica de mercado, resguardando os princípios de concorrência e livre escolha do usuário, os mecanismos de regulação, a solidariedade financeira entre ricos e pobres e a redefinição das funções do Estado $(12,23)$.

Partindo do princípio da separação entre serviços individuais, que são considerados bens privados e estão a cargo do mercado, e coletivos, que são bens públicos e estão sob os cuidados do Estado, o sistema de saúde foi estruturado em dois regimes (contribu- 
tivo e subsidiado), com acesso regulado pelo governo, que realiza inquéritos anuais para classificar o nível de renda e das necessidades da população cadastrada no Sistema de Identificação de Beneficiários de Subsídios do Estado (SISBEN). A base de sustentação financeira do sistema é a contribuição dos trabalhadores (4\% de cada salário recebido) e dos patrões ( $8 \%$ sobre cada salário pago). No regime contributivo, o Plano Obrigatório de Serviços (POS) é garantido aos empregados que recolhem o suficiente para cobrir os benefícios. No regime subsidiado, o governo complementa os custos da assistência do segurado de baixa renda que obtém um pacote de serviços equivalente a $70 \%$ do POS contributivo. O Estado assume ainda a cobertura das ações não-POS e a assistência da população não filiada mediante um critério de prioridade no atendimento a grupos vulneráveis como crianças, pobres e portadores de doenças graves (12).

A gestão do sistema de saúde colombiano é descentralizada para a esfera departamental que contrata empresas promotoras de saúde (EPS) e administradoras de regime subsidiado (ARS). Cada empresa recebe um repasse de recursos correspondente ao volume de unidades de pagamento por captação (UPC) calculado com base no total de seus filiados. Para execução da assistência, as operadoras de seguro saúde contratam instituições prestadoras de serviços (IPS). Dentre elas, estão as empresas sociais do Estado (ESE), que são instituições públicas responsáveis pelo atendimento de primeiro e segundo nível e pelas ações de saúde coletiva.

O financiamento do sistema de saúde se dá pelo Fundo de Solidariedade e Garantia, que é subdividido em quatro contas: a) a conta Compensação (COMP), destinada ao pagamento do regime contributivo, que é formada pela contribuição dos segurados; b) a conta Solidariedade (SOLIDAR), destinada ao regime subsidiado e composta por um percentual da contribuição dos afiliados ao regime contributivo que recebem acima de quatro salários mínimos e por repasses governamentais; c) a conta Promoção da Saúde e Prevenção de Enfermidades (PS-PE), composta por 0,50\% das UPC pagas às EPS e por aportes do governo para a saúde coletiva; d) a conta Enfermidades Catastróficas (CATAST), que financia o custo de doenças crônicas e de alto risco não incluídas no POS (12).

Peru

O sistema de saúde peruano também passa, neste momento, por um amplo reordenamento que decorre da aprovação da "Ley Marco de Aseguramiento Universal en Salud" pelo Congresso Nacional em 30 de março de 2009 (24). A lei estabelece a cobertura obrigatória de seguro saúde a toda a população e para efetivar esse direito criou o Plano Essencial de Assistência à Saúde (PEAS), provendo a base legal para a sua supervisão e gestão (25).

O PEAS contempla a assistência integral a um grupo de doenças consideradas mais relevantes, de acordo com o perfil epidemiológico do país e respeitando as realidades regionais. Na etapa de implan- tação foram selecionados $45 \%$ da morbimortalidade que afeta a população, dando prioridade a três regiões do país (o Departamento de Loreto não está incluído entre elas) e mais quatro redes de saúde que receberam planos pilotos. Já está prevista, para as fases seguintes, a ampliação do número de regiões contempladas e do percentual de carga de doenças cobertas (26).

Segundo Castro (27), essa lei não atende aos propósitos que anuncia de universalização do acesso, porque, entre outras razões, não altera a segmentação do sistema de saúde peruano, apenas propõe a unificação operacional das diversas estruturas que são paralelas e autônomas. Atualmente existem cinco subsistemas que atendem à população: a) o Ministério da Saúde (MINSA), que abrange o Seguro Integral de Saúde (SIS); b) o Seguro Social em Saúde (ESSALUD) adstrito ao Ministério do Trabalho; c) a rede assistencial das forças armadas (Marinha, Aeronáutica e Exército), que atende o Ministério da Defesa; d) o subsistema da Polícia Nacional do Peru, que abrange o Ministério do Interior; e e) o setor privado, formado por entidades prestadoras de serviços de saúde, seguradoras privadas, clínicas e organizações da sociedade civil (28). Em todo o Peru, a parcela da população à margem dos seguros de saúde chega a $58 \%$, o que representa aproximadamente 16 milhões de habitantes. Do restante, $20 \%$ são cobertos pelo ESSALUD, $18 \%$ pelo SIS e $4 \%$ pelas forças armadas e pelo setor privado (25).

O financiamento do sistema de saúde peruano sofrerá mudanças consideráveis com a implantação da Ley Marco de Aseguramiento Universal en Salud. Na situação anterior, havia diversos fundos autônomos que mantinham cada segmento de seguro. A norma atual prevê a criação de um fundo de asseguramento de saúde de caráter público para viabilizar o acesso ao seguro subsidiado e obriga todas as seguradoras a constituir um fundo solidário que possa garantir o PEAS a todos os seus afiliados. Para administrar os fundos solidários foram instituídas as Administradoras de Fundos de Asseguramento de Saúde, que serão supervisionadas pela Superintendência Nacional de Asseguramento de Saúde $(28,29)$.

\section{Análise comparada dos sistemas de saúde da tríplice fronteira Brasil/Colômbia/Peru}

$\mathrm{Na}$ análise dos sistemas de saúde da região andina e Caribe, Gómez-Camelo (10) identifica diferentes desenhos organizacionais classificados como: SNS, sistema segmentado e sistema baseado no asseguramento. Na maioria dos casos, tanto as reformas dos anos 1990 quanto as atuais tendem para o asseguramento universal de um pacote de serviços básicos, com provisão mista (público-privada), gestão pública do financiamento e regulação estatal. A autora conclui ainda que a comparação dos diversos modelos de gestão permite identificar a configuração externa e interna, as similitudes e diferenças e as convergências e divergências dos sistemas de saúde. A tabela 2 apresenta uma tipologia dos sistemas de saúde brasileiro, colombiano e peruano, comparando as suas 
TABELA 2. Comparação entre os sistemas nacionais de saúde dos países que formam a tríplice fronteira Brasil/Colômbia/Peru

\begin{tabular}{|c|c|c|c|}
\hline \multirow[b]{2}{*}{ Componentes } & \multicolumn{3}{|c|}{ Características dos sistemas de saúde } \\
\hline & Brasil & Colômbia & Peru \\
\hline $\begin{array}{l}\text { Financiamento } \\
\text { (comprometimento de recursos } \\
\text { públicos com a saúde) }\end{array}$ & $\begin{array}{l}\text { O SUS tem como fonte de } \\
\text { financiamento os recursos } \\
\text { federais oriundos da arrecadação } \\
\text { fiscal e das contribuições para a } \\
\text { seguridade social que somados às } \\
\text { contribuições sociais e convênios } \\
\text { internacionais formam o Fundo } \\
\text { Nacional da Saúde. Nos estados e } \\
\text { municípios uma parte dos fundos } \\
\text { vem do orçamento próprio e outra } \\
\text { dos repasses das instâncias }\end{array}$ & $\begin{array}{l}\text { O SGSSS é subdividido em dois } \\
\text { regimes: contributivo e subsidiado. } \\
\text { As fontes de financiamento são } \\
\text { os recursos federais oriundos } \\
\text { da arrecadação de impostos e } \\
\text { das cotas de contribuição dos } \\
\text { assegurados no regime contributivo } \\
\text { que formam o Fundo Solidário de } \\
\text { Garantia. }\end{array}$ & $\begin{array}{l}\text { O sistema de saúde é segmentado. } \\
\text { Os recursos federais oriundos da } \\
\text { arrecadação de impostos financiam } \\
\text { o Seguro Integral de Saúde (SIS) } \\
\text { do MINSA; as contribuições de } \\
\text { segurados financiam o Seguro } \\
\text { Social da Saúde (ESSALUD) e } \\
\text { das forças armadas e policiais do } \\
\text { Peru, enquanto o setor privado é } \\
\text { financiado pelo desembolso direto } \\
\text { dos segurados. }\end{array}$ \\
\hline
\end{tabular}

Relação entre financiamento e prestação de serviços (forma de participação do Estado na oferta e demanda)

\section{Acesso} (universalidade) superiores.

Os fundos de saúde são estruturados nas três esferas de governo (federal, estadual, municipal). A gestão é descentralizada para a esfera municipal. Repasse de recursos fundo a fundo ou por pagamento de serviços diretamente aos prestadores. A oferta de serviços é pública com demanda complementar contratada do setor privado. A remuneração dos prestadores se dá por procedimentos realizados.

Acesso universal a todos os serviços sem distinguir usuário. Ingresso no sistema pelo princípio da cidadania.
Cobertura assistencial pública (integralidade)
Cobertura integral assegurada a todo cidadão em todos os níveis de atenção à saúde (promoção, proteção e recuperação).
O governo federal disponibiliza orçamento para financiar a saúde e cada departamento é certificado para a gestão do sistema. As Empresas Promotoras de Saúde administram a assistência contratando instituições prestadoras de serviço (IPS) públicas e privadas. Empresas sociais do Estado (ESE) prestam serviços às EPS concorrendo com outras IPS privadas.

Seguro financiado com recursos públicos garante o Plano Obrigatório de Saúde (POS) para todo assegurado do regime contributivo ou subsidiado. $O$ governo assume a cobertura da assistência à parcela da população que não se encontra afiliada.
A gestão dos sistemas públicos de saúde é centralizada na esfera federal do governo. As redes de serviços MINSA e ESSALUD são paralelas e autônomas na estrutura e funcionamento. $\mathrm{O}$ setor privado é contratado pelos seguros públicos para atender a demanda complementar.
A rede MINSA atende a população de baixa renda, a rede ESSALUD atende aos servidores públicos e profissionais liberais, a rede militar atende a marinha, aeronáutica, exército e polícia nacional, os seguros privados atendem por desembolso direto.. $\mathrm{O}$ acesso em todos os seguimentos depende de afiliação que garante o Plano Essencial de Assistência à Saúde (PEAS).

Plano Obrigatório de Saúde (POS) assegurado a todos os cidadãos. Ações não-POS e assistência dos não afiliados são assumidas pelo governo departamental através das ESE e da contratação de prestadores privados.
Desde março de 2009 a Ley Marco do Aseguramiento Universal prevê a cobertura de assistência integral às doenças prioritárias através do Plano Essencial de Assistência à Saúde (PEAS).

Fonte: elaborada pelos autores.

características, para poder avaliar as possibilidades de se construir um sistema único de saúde na tríplice fronteira.

Com relação ao financiamento, García (15) considera que houve uma tendência de redução dos investimentos em toda a América Latina, implicando em cortes no orçamento e redução das contribuições sociais que dão sustento à seguridade social. Todavia, essa não é uma característica comum a todos os países que formam a tríplice fronteira amazônica (tabela 2). No caso do Brasil, por exemplo, o debate gira em torno da adoção de fontes de recursos definidas, definitivas e suficientes para ampliar a capacidade de oferta de serviços pelo setor público. Nesse sentido, a Emenda Constitucional 29 (EC29) e outros dispositivos apresentam avanços como definição de percentu- ais mínimos do orçamento a ser investidos na saúde por cada esfera de governo e critérios para estipular o que é de fato investimento em saúde $(30,31)$. Nos casos da Colômbia e do Peru, o sistema de saúde tende a ser mais afetado pelas crises econômicas que provocam flutuações no volume de receitas por se basearem na lógica do asseguramento. Mesmo assim, considerando o ano de 2008, o Brasil investiu 7,90\% e a Colômbia $7,30 \%$ do produto interno bruto (PIB), posicionando ambos acima da média continental de $7,10 \%$ do PIB. O Peru, por sua vez, ocupou a última posição do continente com investimentos na ordem de 4,30\% do PIB. Esse dado é importante, tendo em vista a relação direta entre o desempenho dos indicadores de saúde e o montante de recursos investidos no setor $(10,25,29)$. 
Na relação entre financiamento e provisão, García (15) identifica como tendências: o financiamento das demandas; maior autonomia orçamentária e financeira dos serviços públicos; e a integração dos serviços públicos e privados de saúde. Estas características estão presentes na Colômbia e no Peru, com ligeiras variações entre os dois modelos que tendem para uma uniformização depois que o Peru adotou o PEAS, nos moldes do POS colombiano (tabela 2). Diferentemente, no Brasil a esfera pública financia, regula e atua na provisão de serviços, enquanto o setor privado, apesar da sua magnitude, tem apenas uma participação complementar no sistema de saúde.

Quanto à focalização do financiamento público, García (15) ressalta que tende para as seguintes formas: oferta gratuita apenas para a população carente, como se deu no Chile; oferta para a população assegurada com cobrança dos serviços prestados, como se deu no Uruguai; ou seguro subsidiado com recursos públicos, a exemplo do que acontece na Colômbia com o POS e daqui para a frente no Peru depois da implantação do PEAS. No Brasil, a identificação de parcelas vulneráveis serve apenas ao propósito de garantir o princípio da equidade, que justifica o investimento e a cobertura de ações que dão prioridade à redução dos efeitos das diferenças sociais, étnicas, de gênero ou raciais. A discriminação nesse caso é positiva.

A última característica identificada por García (15) como uma tendência geral dos sistemas de saúde é o racionamento da assistência médica, com financiamento público apenas de uma cesta básica de serviços de saúde, justamente o que encontramos na Colômbia e no Peru, onde o POS e o PEAS implicam num contingenciamento da cobertura. Novamente há nuanças no modelo colombiano, o qual distingue os usuários pelo regime de seguro a que têm direito e, a partir daí, discrimina o padrão de cobertura a que fazem jus no pacote de benefícios obrigatório. Enquanto isso, o Peru, que antes dispunha de uma integralidade que era prevista, mas não assegurada no sistema público, agora passa a dispor de um asseguramento previsto e regulado, porém, restrito a um pacote de doenças consideradas prioritárias.

\section{Estrutura e organização dos sistemas de saúde na tríplice fronteira Brasil/Colômbia/Peru}

Brasil

No Brasil, o governo regula a atividade de assistência e mantém um Cadastro Nacional dos Estabelecimentos de Saúde (CNES), obrigatório a todos os prestadores de serviços, que podem ser públicos ou privados, com ou sem fins lucrativos, ou atendendo em regime ambulatorial ou de internação. Dentro deste critério estão incluídos: postos e centros de saúde; clínicas; prontos-socorros; laboratórios; secretarias de saúde; hospitais; e unidades mistas, que são um tipo de estabelecimento destinado à atenção integral, nas especialidades básicas, que conta com uma unidade de internação agregada e sob administração única, podendo atender urgência e emergência.
No Alto Solimões existem 32 unidades de saúde que prestam atendimento de nível ambulatorial e hospitalar. Benjamin Constant é o município com o maior número de unidades (11), seguido por Tabatinga (9), São Paulo de Olivença (4), Tonantins (3), Atalaia do Norte e Santo Antônio do Içá (2) e Amaturá (1). Os tipos mais comuns na região são os centros de saúde $(43,75 \%)$, os postos de saúde $(21,88 \%)$ e as unidades mistas $(18,75 \%)$. Considerando os registros do CNES, a rede assistencial da região é predominantemente pública (96,88\% do total de unidades), havendo apenas um estabelecimento privado (3,12\% do total). No que tange à gestão, a esfera municipal abrange $75 \%$ dos estabelecimentos, contra $18,75 \%$ da esfera estadual que controla seis unidades mistas, 3,13\% da esfera federal que administra o Hospital Geral ligado ao Exército Brasileiro e 3,13\% da esfera privada que responde pela Clínica Odontomédica, localizada em Tabatinga. Se se levar em conta a oferta de leitos hospitalares e a presença de profissionais médicos, cirurgiões dentistas e enfermeiros, cinco dos sete municípios da região deixaram de atingir os valores recomendados pela Organização Mundial da Saúde (OMS) em todos esses itens, comprovando o nível elevado de carência de infraestrutura e recursos humanos na fronteira. As seis unidades mistas do Alto Solimões, somadas ao Hospital Militar de Tabatinga, dispõem de 224 leitos que correspondem a 1,22/1 000 habitantes da região, bem abaixo da média nacional do Brasil situada na casa de 3,60/1 000 e também abaixo do recomendado pela OMS, que é de 4,50/1 000. Em relação a médicos, nenhum município do Alto Solimões alcança a taxa de 0,80/1000 habitantes recomendada pela OMS. Somente no caso de cirurgiões-dentistas, Benjamin Constant e Tabatinga atingiram a cota mínima sugerida de 0,18/1 000 e na enfermagem somente Benjamin Constant superou o patamar de 0,44/1 000 exigido pela OMS. A carência é maior ainda em relação ao nível de capacitação profissional. Entre os 45 médicos que atuam na região, as especialidades mais frequentes são gineco-obstetrícia (15 profissionais em cinco municípios) e cirurgia geral (11 profissionais em seis municípios). Os "médicos de família" somam apenas sete profissionais distribuídos por três municípios, apesar da estratégia nacional de estruturação da atenção básica estimular a implantação do Programa de Saúde da Família, que tem sido fundamental para a efetividade das ações de primeiro e segundo nível. A falta de especialistas de outras áreas compromete a qualidade da assistência de média e alta complexidade na região $(32,33)$.

\section{Colômbia}

Na Colômbia, a estrutura e organização dos serviços de saúde obedecem às diretrizes da Lei 100/1993 que instituiu a descentralização e o asseguramento, mediante afiliação obrigatória a um dos regimes (contributivo ou subsidiado). Para regulamentar o processo de descentralização, a Lei 715/2001 (34) redefiniu os fluxos de recursos financeiros e as competências municipais, responsabilizando a instância 
departamental pela gestão do SGSSS, pela prestação de serviços de primeiro e segundo níveis e pela cobertura de assistência da parcela da população que ainda não se encontra filiada a nenhum dos regimes de seguro saúde (35).

O Amazonas colombiano está certificado pelo Ministério da Saúde como território descentralizado. Sendo assim, assumiu as funções de organização, controle e fiscalização do sistema de saúde e se encarrega de todas as IPS localizadas no departamento (hospitais, centros e postos de saúde), além de ser o prestador direto de serviços nos municípios de Leticia e Puerto Nariño e nos 10 corregimientos. Para atender a esta finalidade, a estrutura organizacional da Secretaria de Saúde Departamental do Amazonas conta com três áreas: a) a área de direção, encarregada da administração dos recursos e a da direção do setor; $b$ ) a área de segurança, vigilância e controle; c) a área de serviços de saúde (prestadores) encarregada do componente da saúde pública e da prestação de serviços de saúde (8).

Segundo levantamento realizado no ano de 2006, apenas 17,04\% da população do Departamento do Amazonas se encontrava filiada ao regime contributivo, enquanto no regime subsidiado a proporção chegava a 58,52\%, os pobres não assegurados representavam $13,27 \%$ e os que não tinham a sua modalidade de filiação identificada atingiam $7,70 \%$ da população. Em Leticia, a parcela assegurada no regime contributivo naquele ano era um pouco mais expressiva $(26 \%)$, enquanto o regime subsidiado se manteve no patamar de $46,30 \%$. Em compensação, mais de um quinto da população $(21,80 \%)$ era classificada como pobre sem cobertura de seguro em plena capital do departamento. A situação piorava em Puerto Nariño, onde o regime contributivo contemplava apenas uma faixa de $2,50 \%$ da população e o regime subsidiado abarcava $80,90 \%$ da população. A mesma situação se repetia nos corregimientos, com $76,10 \%$ da população vinculada ao regime subsidiado, contra apenas $0,59 \%$ que era assegurada no regime contributivo (8).

A existência de uma parcela tão expressiva da população sem acesso ao seguro e de uma baixa afiliação no regime contributivo são tidos como problemas relevantes porque inviabilizam o financiamento do SGSSS. Os registros nacionais revelaram que entre 1999 e 2000 apenas 32\% da população colombiana estavam filiados ao regime contributivo e $22 \%$ ao regime subsidiado. As autoridades de saúde advertiam, naquela ocasião, que cerca de $13,50 \%$ dos filiados ao regime contributivo poderiam ser repetidos, dada à baixa qualidade do sistema de informação das EPS, indicando que o controle público das operadoras de seguro seria a principal atividade da gestão que poderia garantir o acesso aos serviços de saúde (12).

No departamento do Amazonas há três ARS autorizadas a operar em Leticia (CAPRECOM, MALLAMAS, SELVASALUD), duas em Puerto Nariño (CAPRECOM e SELVASALUD) e três nos corregimientos departamentais (MALLAMAS, HUMANAVIVIR e SELVASALUD). Para administrar o regime contribu- tivo, quatro EPS estão autorizadas (SALUDCOOP, ISS, CAPRECOM e SANITAS) (36).

No que diz respeito à prestação de serviços, a rede pública do Amazonas conta com dois hospitais: o Hospital San Rafael de Leticia, que é a única instituição prestadora de segundo nível de atenção no departamento, e outro hospital que se localiza no município de Puerto Nariño e presta serviços de primeiro nível. Existem ainda sete centros de saúde localizados nos corregimientos que também prestam serviços de primeiro nível. Esses centros de saúde dependem financeira e administrativamente do governo departamental para atender aos colonos e indígenas, dando cobertura à rede de 82 postos de saúde espalhados pelos corregimientos e contando, para isso, com um sistema de comunicação por rádio que é utilizado em caso da necessidade de remoção de pacientes (8).

Os procedimentos de baixa complexidade são oferecidos em toda a rede pública. Nesse rol estão incluídos a consulta ambulatorial externa por médico generalista, atividades odontológicas, procedimentos de imunização e exames citológicos, consultas de urgência e emergência, hospitalização e partos normais. A média complexidade existe apenas no Hospital São Rafael de Leticia, que conta com especialidades nas áreas de clínica cirúrgica, ginecologia e obstetrícia, medicina interna, pediatria, anestesiologia, ortopedia e oftalmologia. Outras subespecialidades são ofertadas esporadicamente, em parceria com instituições nacionais, e as demandas de alta complexidade são todas resolvidas com encaminhamentos para os grandes centros urbanos de Bogotá, Neiva e Villavicencio (36).

Fora de Leticia, a infraestrutura disponível na rede pública é bastante diversificada. No Hospital de Puerto Nariño há equipamentos para atendimento de urgências e emergências e recursos laboratoriais até para serviços de alta complexidade. Em toda a rede pública localizada em Puerto Nariño e nos 10 corregimientos departamentais existem 48 leitos de internação. A rede de assistência à saúde no departamento do Amazonas é complementada pelas IPS localizadas em Leticia, sendo que uma delas oferece serviços que vão até o terceiro nível e outra que atende urgências de baixa complexidade e consultas externas $(8,36)$. Somando os serviços públicos (3), privados (17) e consultórios profissionais (19) aos estabelecimentos de outras modalidades (3), Leticia conta com 42 IPS que possuem 85 leitos de internação, sendo 42 no Hospital San Rafael, 27 na Fundação Clínica Leticia e 16 na Clínica de Especialistas.

Em resumo, do ponto de vista da gestão o sistema de saúde do Amazonas é público e sob o comando da esfera departamental. Quanto ao asseguramento, há empresas públicas e privadas que atuam como operadoras do regime contributivo (EPS) ou do regime subsidiado (EPS-S ou ARS). Na administração dos serviços de saúde estão as IPS, que também podem ser públicas (ESE) ou privadas, atuando em um ou mais níveis de assistência. No departamento do Amazonas há duas IPS públicas: a ESE Hospital San Rafael de Leticia e a ESE Amazon Vida que administra 
os centros de saúde e postos de saúde dos corregimientos e o hospital local de Puerto Nariño $(8,36)$.

Dadas as características do sistema de saúde colombiano, no qual a rede básica é de competência departamental e a assistência individual é privatizada, ao gestor municipal restam apenas as funções de controlar o processo de identificação do usuário, manter atualizado o banco de dados do SISBEN e estimular a filiação da população, além da execução de ações de saúde de alcance coletivo. Para essa última finalidade a Lei 1122 de janeiro de 2007 introduziu ajustes no SGSSS que buscam promover a universalização do acesso, o equilíbrio entre os atores do sistema e a melhoria na prestação de serviços de saúde, com fortalecimento das ações de promoção e prevenção, as quais foram agrupadas em um Programa de Saúde Pública de caráter obrigatório, sob a responsabilidade do setor público e com destinação de recursos previamente garantida no conjunto das obrigações das seguradoras (35). Trata-se de uma tentativa de ampliar a oferta seguindo uma linha universalizante, no interior de um sistema estruturado sob a lógica do asseguramento, que opera com regras de mercado e cuja finalidade precípua é financiar a demanda (37).

\section{Peru}

No Peru os serviços de saúde são organizados em subsistemas correspondentes aos segmentos de seguro saúde (38). Desta forma, a população de Loreto dispõe de quatro redes de assistência - com estruturas independentes, corpo de funcionários próprio e autonomia administrativa - cada qual dirigida a uma parcela de assegurados. Ao todo, são 347 estabelecimentos distribuídos entre as sete províncias do departamento. A rede pública do MINSA é a mais importante, contando com 319 unidades (91,93\%). A rede militar é a segunda, contando com 16 unidades $(4,61 \%)$. A rede ESSALUD e a rede privada, cada uma com 6 unidades (1,73\%), ficam em terceiro lugar (9). Em contraste com a situação da Colômbia, onde o setor público se responsabiliza formalmente pela atenção básica, no sistema de saúde peruano todo usuário tem direito a assistência integral, que deve ser ofertada pelo plano de seguro a que está filiado; no entanto, as características da rede de saúde demonstram que predominam os serviços de primeiro e segundo níveis, havendo apenas uma unidade de terceiro nível em todo o departamento de Loreto.

A denominação dos estabelecimentos de saúde peruanos segue a tipologia de cada segmento de prestação de serviços. No sistema MINSA, as unidades básicas são identificadas como do tipo posto de saúde (PS I e PS II) e centro de saúde (CS I e CS II), que diferem entre si pelo tipo de profissional disponível e pelo regime de trabalho. Os PS I têm médicos e enfermeiros, já os PS II têm técnico de enfermagem e técnico sanitário. No caso do CS I e do CS II, a diferença decorre da presença de sala de internação. $\mathrm{O}$ tipo que usualmente conta apenas com técnicos de enfermagem é mais frequente, representando 77,01\% das unidades de saúde de Loreto. Já o tipo que conta com equipe completa de profissionais chega a $3 \%$ de toda a rede. Na rede ESSALUD, as unidades são do tipo centro de saúde (CS) e hospital. O sistema militar classifica as suas unidades como: postos de saúde (PS) e hospitais. O setor privado utiliza a terminologia clínica e hospital (9).

De acordo com os dados de 2007, fornecidos pela Direção Regional de Saúde de Loreto (DIRESA), o sistema MINSA concentra $99,06 \%$ da sua rede no primeiro nível de assistência. A rede militar tem $81,25 \%$ das unidades no primeiro nível e $18,75 \%$ no segundo. A rede ESSALUD conta com $66,76 \%$ no primeiro e $33,33 \%$ no segundo nível. O setor privado concentra todas as suas unidades no segundo nível de assistência. Esses dados revelam o grau de concentração da assistência de maior complexidade nos grandes centros urbanos, principalmente em Lima, a capital nacional (9).

O sistema MINSA estrutura-se a partir das unidades básicas que estão distribuídas de acordo com a facilidade de comunicação e de referência dos pacientes. Assim, um conjunto de centros e postos de saúde conforma uma microrrede e um conjunto de microrredes conforma uma rede. Administrativamente o departamento de Loreto está dividido em duas redes: a rede Alto Amazonas, que agrega duas microrredes, e a rede Loreto, que contempla 10 microrredes. Cada microrrede corresponde a uma das regiões definidas pela divisão político-geográfica do departamento. Assim, as duas microrredes de Marañon e Huallaga formam a rede Alto Amazonas, enquanto a rede Loreto agrega as microrredes de Requena, Ucayali, Loreto e Ramón Castilla, além daquelas localizadas na província de Maynas que, por ser a mais extensa e mais populosa, agrega seis microrredes: Putumayo, Mazán, Napo, Belén, Iquitos Norte e Iquitos Sur. Estas duas últimas concentram, cada qual, mais de 100 mil habitantes, enquanto as de Napo e Putumayo não ultrapassam 25 mil habitantes (9).

A oferta de serviços e a disponibilidade de profissionais de saúde é um problema grave em todo o departamento de Loreto, onde nenhuma província alcança o número mínimo de médicos, enfermeiros, odontólogos e leitos hospitalares recomendado pela OMS. A distribuição também não é equitativa, pois a região de fronteira constitui o espaço com maior nível de carência. Dos nove hospitais existentes em Loreto, cinco deles estão localizados em distritos urbanos das províncias de Maynas e Alto Amazonas, enquanto cerca de 90 mil pessoas residentes nos 12 distritos fronteiriços com Brasil, Colômbia e Equador contam com 74 unidades básicas, das quais apenas duas são centros de saúde e o restante são postos que dispõem apenas de pessoal de nível técnico.

\section{Possibilidades de integração dos sistemas de saúde na tríplice fronteira amazônica}

Há convergências entre os sistemas de saúde dos três países fronteiriços quanto à participação do Estado no financiamento, na regulação e na prestação 
de serviços, mas também há discrepâncias que dificultam uma atuação compartilhada.

Enquanto no Brasil o SUS constitui um Sistema Nacional de Saúde de finalidade pública com provisão mista de serviços (23), os sistemas de saúde da Colômbia e do Peru têm como traços comuns o financiamento pelo asseguramento, o acesso pela filiação e o provimento de serviços através do subsídio à demanda (tabela 2). No caso da Colômbia, o POS prioriza uma cesta básica de serviços e no Peru o PEAS prevê uma assistência focalizada pelo nível de risco (39). Nas duas situações, as autoridades sanitárias até procuram exigir das operadoras um gasto mínimo com ações de saúde pública de alcance coletivo, sem que isso reverta na integralidade, que depende de uma ação articulada do sistema de saúde com outros setores sobre os determinantes da condição de vida da população (15).

Segundo Jaramillo (37), a substituição do subsídio da oferta pelo subsídio da demanda tem como objetivos enunciados a concentração dos benefícios do financiamento público nas camadas mais pobres e a melhoria da produtividade dos serviços públicos, incentivando a competitividade com o setor privado pela preferência do consumidor. Para tanto, adota como estratégia a separação entre a administração da demanda e o provimento de serviços, tendo como resultado a configuração de um mercado de serviços médico-hospitalares regido pela lei da oferta e da procura, que torna incompatível tanto a regulação da eficácia dos gastos quanto a garantia do acesso e a cobertura integral.

\section{CONCLUSÃO}

O processo de reforma sanitária ocorrido na América Latina repercutiu no desenvolvimento dos sistemas de saúde e na organização dos serviços na tríplice fronteira Brasil/Colômbia/Peru. O modelo adotado pelo Brasil tem as características de um sistema nacional de saúde de finalidade pública, enquanto na Colômbia e no Peru vigoram sistemas de asseguramento que promovem a segmentação dos usuários, apesar da adoção de planos obrigatórios de assistência com níveis variados de contingenciamento da cobertura. Os três países adotam modelos que mesclam o público e o privado na provisão de serviços com diferentes níveis de regulação por parte do Estado.

Nas condições em que se dá a estruturação da rede e a gestão da saúde na tríplice fronteira Brasil/ Colômbia/Peru, o setor público predomina como provedor dos serviços básicos tornando possível uma intervenção articulada, com oferta organizada e ações programáticas de saúde no primeiro e segundo níveis de atenção.

As características próprias de cada um dos sistemas de saúde implicam em dificuldades operacionais de integração, principalmente na assistência de média e alta complexidade, porque, no sistema de saúde da Colômbia e do Peru, onde as instituições privadas têm um peso maior no atendimento individual, as condições de acesso e cobertura se dão com graus elevados de diferenciação entre usuários.

Este fato torna mais complexo o desafio da construção de um sistema único de saúde na fronteira, visando garantir a universalidade do acesso, a equidade no atendimento e a integralidade na assistência. Para solucionar essa dificuldade, poderia ser estruturado na região um fundo único de saúde, de caráter público, com recursos oriundos de fontes nacionais e da cooperação internacional, que tivesse uma gestão trinacional e pudesse financiar o acesso universal e a cobertura integral dos serviços de saúde, independentemente da natureza pública ou privada do prestador. Essa poderia ser a base de um sistema único de saúde da fronteira.

\section{ABSTRACT}

\section{Comparative analysis of health systems on the triple border between Brazil, Colombia, and Peru}

The objectives of this article are to describe the health sector reform process in Latin American countries and its impact on the health systems of Brazil, Colombia, and Peru; analyze the structure of the national health systems of these bordering nations; and assess the constraints and potential for integrating the local health systems along the triple Amazon border. Using official documents and databases, we analyzed health system development and the structure of the services network in this segment of the border located in the heart of the Amazon region. Comparison of the three countries confirmed that Brazil's model has the characteristics of a national public health system, while the Colombian and Peruvian models emphasize insurance systems, with the adoption of compulsory health care plans, the segmentation of users, and limitations on coverage. The three countries are similar in their adoption of a public-private mix in service delivery, but they differ in the degree of access and type of State regulation. We concluded that the characteristics of the health systems in the three countries make the challenge of building a unified health system capable of guaranteeing universal access, equity in care, and comprehensive care a complex undertaking. However, despite the differences identified, we can state that the weight of the public sector in the basic care network makes it possible to foresee integration of the local health systems to provide an organized supply of programmatic health actions at the primary and secondary level of care.

Key words: health systems; health services administration; health care reform; border health; Brazil; Colombia; Peru; South America. 


\section{REFERÊNCIAS}

1. Peiter PC. Geografia da Saúde na Faixa de Fronteira Continental do Brasil na Passagem do Milênio. Tese de doutorado. Rio de Janeiro: UFRJ/IGEO/ PPGG; 2005.

2. Brasil. Ministério da Integração Nacional. Secretaria de Programas Regionais. Proposta de Reestruturação do Programa de Desenvolvimento da Faixa de Fronteira. Brasília; 2005.

3. Viacava F, Almeida C, Caetano R, Fausto M, Macinko J, Martins M, et al. Uma metodologia de avaliação do desempenho do sistema de saúde brasileiro. Cienc Saude Coletiva. 2004;9(3):711-24.

4. World Health Organization. The World Health Report: Health System: Improving Performance. Genebra: OMS; 2000.

5. Mendes EV. Os sistemas de serviços de saúde: o que os gestores deveriam saber sobre essas organizações complexas. Escola de Saúde Pública do Ceará. Fortaleza; 2002.

6. Souza M. História da Amazônia. Manaus: Editora Valer, 2009.

7. Instituto Brasileiro de Geografia e Estatística. Sistema IBGE de recuperação automática: censo demográfico e contagem populacional. http://www.sidra. ibge.gov.br/ Acessado em 26 de março de 2009.

8. Colombia. Gobernación del Amazonas/ Secretaria de Salud Departamental. Estudio para la Organización de la Prestación de Servicios de Salud de Primer Nivel y su Articulación a la Red de Prestación de Servicios del Departamento. Leticia; 2007.

9. Perú. Dirección Regional de Salud Loreto. Análisis de Situación de Salud Región Loreto, Año 2007. Iquitos; 2008.

10. Gómez-Camelo D. Análisis comparado de los sistemas de salud de la región Andina y el Caribe. Rev Salud Publica [online]. 2005;7(3):305-16. ISSN 01240064.

11. Vergara C. El contexto de las reformas del sector de la salud. Rev. Panam Salud Publica. 2000;8(1):7-12.

12. Hernández M. Reforma sanitaria, equidad y derecho a la salud en Colombia. Cad Saude Publica. 2002;18(4):991-1001.

13. Björkman JW. Reformas de saúde em perspectiva comparada: uma questão sem fim ou uma busca improvável. Cien Saude Coletiva. 2009;14(3):763-70.

14. Guzmán Urrea, MP. Deficiencias en los diagnósticos de las reformas sanitarias de los años noventa en Amé- rica Latina. Rev Panam Salud Publica. 2009;25(1):84-92.

15. García GG. Las reformas sanitarias y los modelos de gestión. Rev Panam Salud Publica. 2001;9(6):406-12.

16. Brasil. Constituição da República Federativa do Brasil. Brasília: Senado Federal; 1988.

17. Brasil. Lei 8.080/90. Brasília: Diário Oficial da União; 1990a.

18. Brasil. Lei 8.142/90. Brasília: Diário Oficial da União; 1990b.

19. Escorel S, Nascimento DR, Edler FC. As origens da reforma sanitária e do SUS. Em: Lima NT, Gerschman S, Edler FC, orgs. Saúde e democracia: história e perspectivas do SUS. Rio de Janeiro: Editora Fiocruz; 2005.

20. Cunha JPP e Cunha RE. Sistema Único de Saúde - Princípios. In: Campos FE, Tonon LM, Oliveira Júnior M. Caderno Saúde, vol 1. Coopmed: Belo Horizonte; 1998.

21. Menicucci TMG, Marques AMF, Oliveira BR, Fortes FBCTP, Duarte VC. Regionalização da atenção à saúde em contexto federativo e suas implicações para a equidade de acesso e a integralidade da atenção. Belo Horizonte: Fundação João Pinheiro; 2008.

22. Colombia. Ley 100/93: Congreso de la República. Bogotá; 1993.

23. Boiniec IL. Sistemas de protección social comparados: Colombia, Brasil y México. Rev Salud Pública. 2002;4(3):203-39.

24. Perú. Congreso de la República. Ley $\mathrm{N}^{\circ}$ 29344: Ley marco de aseguramiento universal en salud. Lima: Congreso de la República; 2009.

25. Diaz L, Vargas J. Ley marco de aseguramiento universal en salud, bajo la lupa. Rev Peru Med Exp Salud Publica. 2009;26(2):236-42.

26. Arce M. Implementación del aseguramiento universal en salud en regiones piloto del Peru. Rev Peru Med Exp Salud Publica. 2009;26(2):218-21.

27. Castro J. Hacia el aseguramiento universal en salud en el Peru. Rev Peru Med Exp Salud Publica. 2009;26(2):232-5.

28. Wilson L, Velásquez A, Ponce C. La ley Marco de Aseguramiento Universal en Salud en Perú: análisis de beneficios y sistematización del proceso desde su concepción hasta su promulgación. Rev Peru Med Exp Salud Publica. 2009;26(2):207-17.

29. Barboza-Tello M. El aseguramiento universal en el Peru: la reforma del finan- ciamiento de la salud en perspectiva de derechos. Rev Peru Med Exp Salud Publica. 2009;26(2):236-8.

30. Ugá MAD, Santos IS. Uma análise da progressividade do financiamento do Sistema Único de Saúde (SUS). Cad Saude Publica. 2006;22(8):1597-609.

31. Cohn A. A reforma sanitária brasileira após 20 anos do SUS: reflexões. Cad Saude Publica. 2009;25(7):1614-19.

32. Camargo Jr KR de et al. Avaliação da atenção básica pela ótica políticoinstitucional e da organização da atenção com ênfase na integralidade. Cad Saude Publica. 2008;24(1):s58-s68.

33. Conill EM. Ensaio histórico-conceitual sobre a Atenção Primária à Saúde: desafios para a organização de serviços básicos e da Estratégia Saúde da Família em centros urbanos no Brasil. Cad Saude Publica. 2008;24(1):s7-s16.

34. Colombia. Ley 715/01: Diario Oficial. Bogotá; 2001.

35. Silva RL. Promoción, prevención, municipalización y aseguramiento en salud, en siete municipios. Rev Salud Publica. 2002;4(1):3658-78.

36. Colombia. Departamento del Amazonas/Alcadía de Leticia. Plan de desarrollo de la mano con la comunidad. Municipio de Leticia 2010-2011. Leticia; 2008.

37. Jaramillo I. Evaluación de la descentralización de la salud y la reforma de la Seguridad Social en Colombia. Gaceta Sanitaria [online]. 2002:16(1):48-53 Acessado em 2 de setembro de 2010.

38. Salaverry O, Cárdenas-Hojas D. Establecimientos asistenciales del sector salud, Peru 2009. Rev Peru Med Exp Salud Publica. 2009;26(2):264-7.

39. Velásquez A. La carga de enfermedad y lesiones en el Perú y las prioridades del plan esencial de aseguramiento universal. Rev Peru Med Exp Salud Publica. 2009;26(2):222-31.

Manuscrito recebido em 11 de março de 2010 Aceito em versão revisada em 17 de setembro de 2010. 